\title{
Experimental Verification of Four Wave Mixing Efficiency Characteristics in a Few Mode Fibre
}

\author{
N. Mac Suibhne ${ }^{(1 \mathrm{a})}$, A.D.Ellis ${ }^{(2, *)}$, F.C. Garcia Gunning ${ }^{(1 \mathrm{~b})}$, S.Sygletos $^{(2)}$ \\ ${ }^{(1)}$ Tyndall National Institute and Department of ${ }^{(a)}$ Electronic Engineering ${ }^{\text {(b) }}$ Physics, University College \\ Cork, Ireland \\ (2) Aston Institute of Photonic Technologies, Aston University, Birmingham, England, \\ * andrew.ellis@aston.ac.uk
}

\begin{abstract}
We introduce two techniques to measure the efficiency of inter mode FWM with respect to intra mode FWM. The first technique allows an estimation of the additional FWM penalty for any given system; the second isolates the contribution of each mode. Measurements are compared to an analytical model showing the FWM signal increases by $\sim 2 d B$ with inter mode phase matching.
\end{abstract}

\section{Introduction}

Recently, it has been confirmed that the maximum capacity of a single mode fibre may be deduced by integrating the quasi phase matched four wave mixing (FWM) efficiency over the signal bandwidth ${ }^{1-3}$, with the distance and bandwidth scaling rules confirmed experimentally ${ }^{4}$. The same calculations will apply in few mode and multi-mode fibres, subject to a suitable scaling factor to account for random mode coupling ${ }^{5,6}$, allowing a preliminary estimation of the capacity limit ${ }^{7}$. However, for sufficiently broadband wavelength division multiplexed systems, inter-mode phase matching is possible ${ }^{8}$. This has been observed experimentally for both cross phase modulation using isolated pulses $^{9}$ and four wave mixing using continuous wave signals ${ }^{10,11}$. However, whereas these experiments confirm the presence of the inter-mode phenomena, quantification of their impact remains elusive.

In this paper, we experimentally confirm both the logarithmic bandwidth dependence of FWM in a few mode fibre and quantify the relative magnitude of intra- and inter-mode FWM through a simple experiment based on FWM between blocks of amplified spontaneous emission (ASE), which resemble closely spaced WDM signals after a few dispersion lengths ${ }^{2}$.

\section{Experimental configuration}

Figure 1 shows the simple experimental configuration used for both measurement techniques. A $30 \mathrm{~dB}$ gain, gain-flattened erbium doped fibre amplifier was used as an ASE source, which was shaped into various rectangular blocks to represent specific wavelength bands of highly dispersed communications signals using a wavelength selective switch (WSS) programmed according to the technique used (see below). These blocks were amplified by a $38 \mathrm{dBm}$ gain-flattened power amplifier. The output of the power amplifier was monitored using the optical spectrum analyzer (OSA) and the WSS was iteratively adjusted to ensure equal power spectral densities across the blocks of ASE. The amplifier current was adjusted, where necessary, to maintain constant spectral power. The centre of the ASE spectrum was attenuated using the WSS to leave a notch of $50 \mathrm{GHz}$ bandwidth.

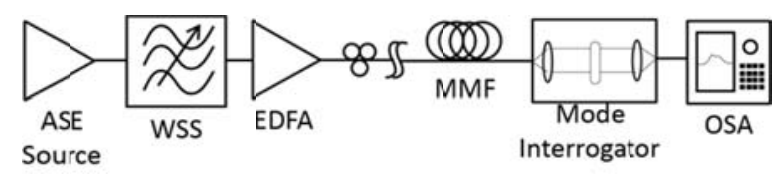

Fig. 1: Schematic diagram of experimental set up (lenses and positioning stages at the ends of few mode fiber are omitted for cliarity).

The high power ASE was launched into a few mode fibre designed to support four LP modes and to have a low differential mode delay at $1550 \mathrm{~nm}^{12}$. We estimate that the launch loss was approximately $1.5 \mathrm{~dB}$ using an offset launch adjusted to maximise mixing between the $L P_{01}$ and $\mathrm{LP}_{11}$ modes, The output the mode interrogator, consisting of coupling lenses, collimator and phase plate, had an excess loss of approximately $5 \mathrm{~dB}$. Outputs from each fibre mode were selected using a phase plate (only one orientation used for $L P_{11}$ ) and the output spectrum was recorded using the same OSA, enabling the output power spectral density and the FWM power generated in the central notch to be established. We performed two similar experiments, as per the illustration in Figure 2, The first used a broadband signal with a $50 \mathrm{GHz}$ notch at the centre, where the total pass-band bandwidth of the ASE block (as illustrated in Fig 2(a)) was varied using the WSS. The FWM signal was monitored by observing the signal generated in the notch This technique was used to show the logarithmic scaling with signal bandwidth and the relative impact of inter and intra mode $\mathrm{FWM}^{4}$. The FWM signal will increase logarithmically until it is sufficiently broad to 
allow intra-mode phase matching where a sudden increase would occur, followed by continuation of the logarithmic growth. This measurement is directly related to the nonlinear noise of a WDM system².

The second technique, (henceforth referred to as narrowband), has a narrower block of ASE, $400 \mathrm{GHz}$ wide with a $50 \mathrm{GHz}$ notch in the centre. A further block of ASE $(200 \mathrm{GHz}$ bandwidth) was then moved in steps of $50 \mathrm{GHz}$ away from the central ASE block.
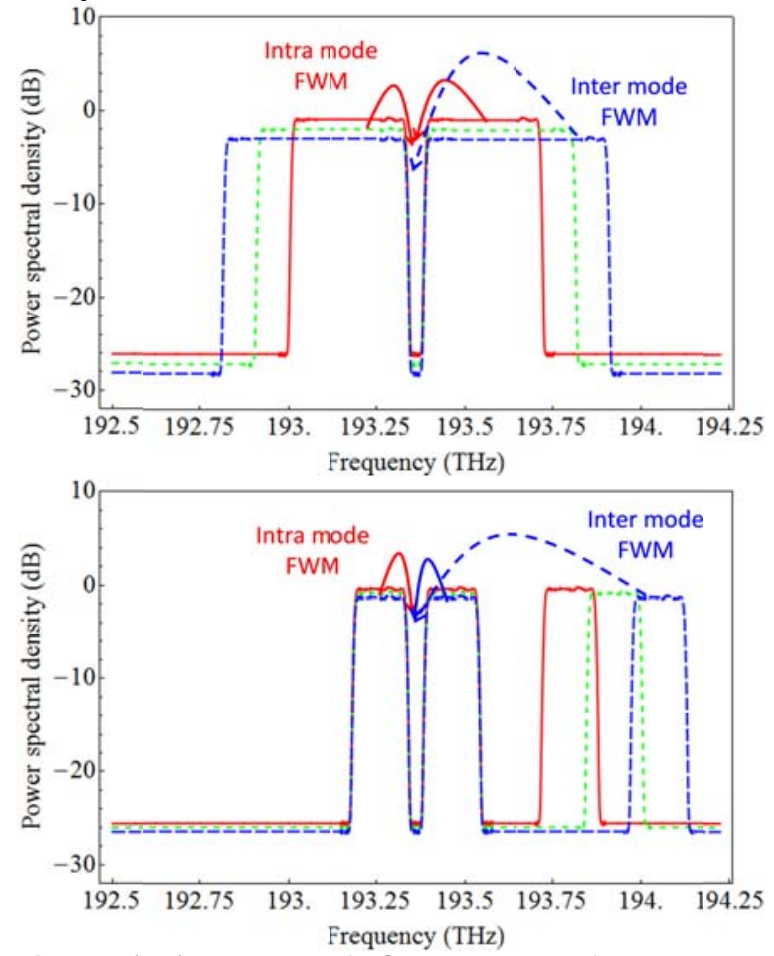

Fig. 2: a (top) Illustration of ASE spectra used for broadband measurement illustrating intra channel four wave mixing processes for narrow bandwidth (solid red arrow) and the potential for phase matched inter mode interactions for sufficiently high bandwidths (dashed blue arrow). b (bottom) illustration of ASE spectra used for narrowband measurement, with a block of ASE with a variable detuning to generate phase matched inter mode FWM.

This technique is used to allow a clear identification of individual inter channel effects and identification of their relative interaction strengths. For this technique the intra mode FWM observed in the notch will have two contributions, one from the ASE close to the notch, and one from the interaction with the remote block, which would be expected to decrease logarithmically. However, we would expect the FWM to be enhanced if the remote block were appropriately detuned from the notch to allow for inter mode phase matching to occur.

\section{Results}

Typical output spectra, zoomed in around the $50 \mathrm{GHz}$ notch, are shown in Fig 3 for both configurations. For the broadband measurement, the wide bandwidth of the broadest test signal and the limited amplifier output power result in a relatively low power spectral density of $1.2 \mathrm{~W} / \mathrm{THz}$ (at the amplifier output), which was maintained as the ASE was varied by reducing the amplifier pump power. A low level of FWM power was generated in the notch, which varied monotonically as a function of the ASE width.
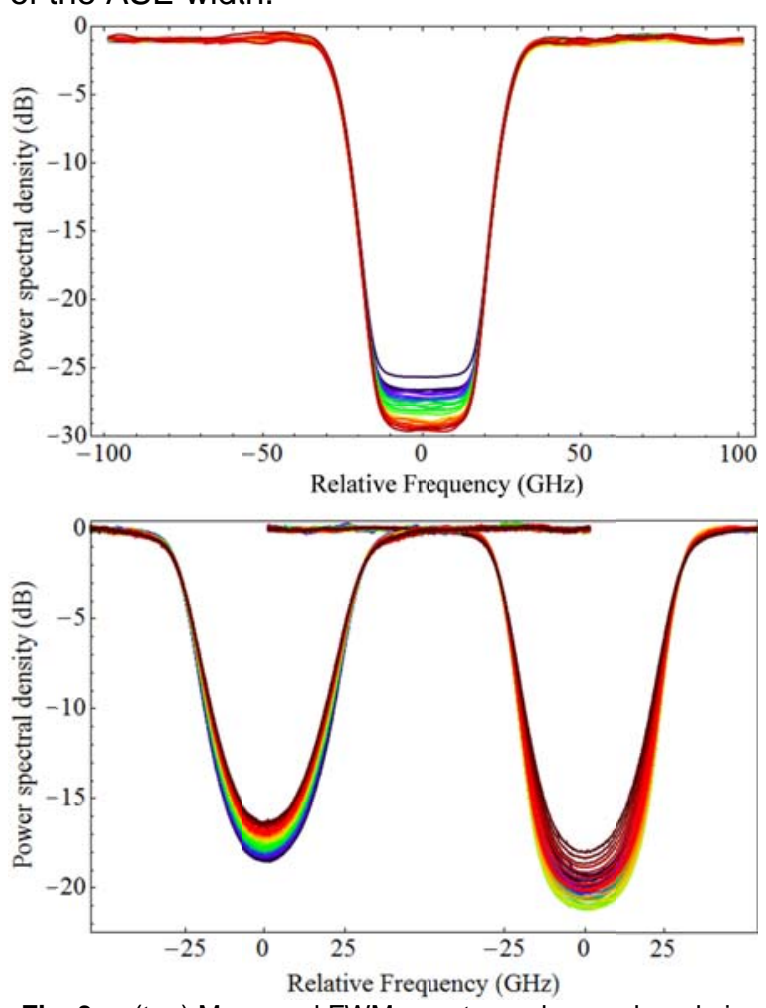

Fig. 3: a (top) Measured FWM spectrum observed mode in the ASE notch for the LP $\mathrm{P}_{01}$ modle (no phase plate) as a function of the ASE bandwidth for the broadband test conditions. $b$ (bottom) the tuning of the additional ASE block in the narrowband condition for negative (left hand curves) and positive (right hand curves) detuning.

For the narrowband test configuration, a constant power spectral density of nearly $10 \mathrm{~W} / \mathrm{THz}$ generated considerably higher levels of FWM within the notch, giving rise to a clear broadening of the edges of the notch. This also led to slight residual errors in the power uniformity even after gain flattening using the WSS, especially for the variable position block. As the additional ASE block is tuned to negative frequencies (left hand curves Fig 3b), the FWM efficiency decreases slightly, again as expected for the measurement configuration. However, for positive tuning (right hand curves Fig $3 b$ ) a more complex evolution is observed, with an initial decrease followed by a clear increase in intensity. We believe that the change in mean FWM efficiency for the two cases is caused by the residual spectral tilt of the ASE signal launched into the fibre. Fig 4 shows the normalized FWM efficiencies recorded for the 
$\mathrm{LP}_{01}$ mode. Good agreement between theory, which predicts logarithmic growth of FWM with bandwidth, and experiment is only possible under the assumption of mode coupling where additional FWM terms are introduced for when the ASE bandwidth exceeds the phase matching frequency offset for a given interaction. From the fitting parameters, we may extract phase matching offsets of $0.7,0.85$ and $1.2 \pm 0.1 \mathrm{THz}$ and peak interaction strengths for the inter mode FWM of around $15 \%$ of the intra mode effects for the first three steps, suggesting that the effective areas for these interactions are up to 2.5 times greater than the effective area of the $\mathrm{LP}_{01}$ mode itself. These values are consistent with the overlap integrals between modes for a four mode fibre. Note that here we ignore the final step at $3 \mathrm{GHz}$. The total FWM signal is approximately $2 \mathrm{~dB}$ greater than expected from intra mode nonlinearity alone.

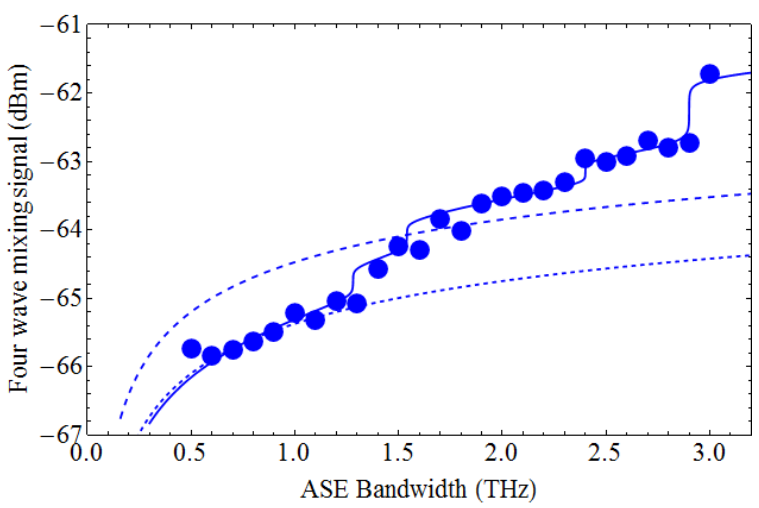

Fig. 4: Measured and calculated FWM power for LP 01 detection (blue) showing experimental data (dots) and theoretical fits with mode coupling (solid line) and without (fitted to whole data set - long dashes, or just initial points short dashes) for the broadband measurement system.

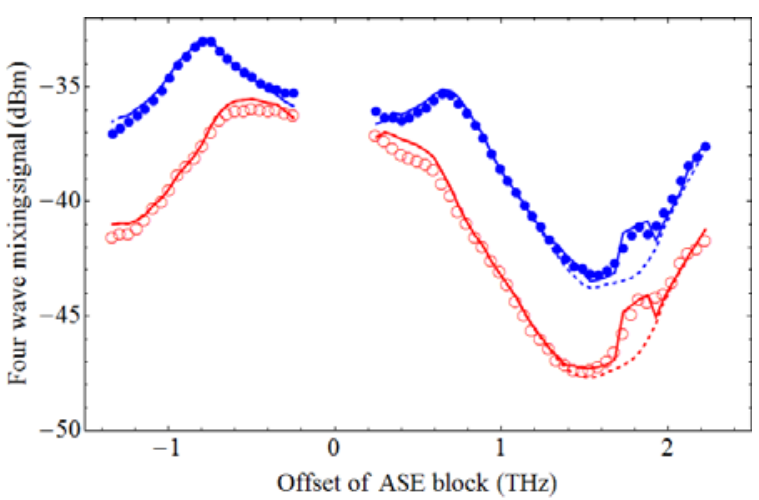

Fig. 5: Measured and calculated FWM power for $L_{01}$ (blue filled) and $L_{11}$ (red open) detection showing experimental data (dots) and theoretical fits with mode coupling (solid lines) and without (dashed lines) for the narrowband measurement system.

Similar agreement between theoretical predictions and experiment are observed for the narrowband measurement, as per Fig 5. Here gain flatness of only $3 \mathrm{~dB}$ was achieved, and so it was necessary to correct the predicted FWM power density for the actual launch power. This was achieved by multiplying the appropriate launch power for each point by an effective length at that wavelength. The effective length was determined from the difference between the input and output spectra. Again, good agreement is reached using the same phase matching offsets as Fig 4. Note that for the $\mathrm{LP}_{01}$ mode, peaks only occur for positively detuned ASE blocks, as expected. Note also that, whilst the frequency offsets are similar, the required inter mode effective areas to fit this data are slightly lower than observed in Fig 4.

\section{Conclusions}

In this paper we have presented two simple techniques to measure the impact of inter mode FWM in few mode fibre based transmission systems. The broadband technique essentially integrates the nonlinear contributions and gives a reliable qualitative estimate of the total FWM power generated at a given signal wavelength. On the other hand, the narrow band measurement isolates the effect of each interaction, and gives further information regarding the sign of the phase matching frequency offset. For this particular fibre, we note that the $2 \mathrm{~dB}$ increase in FWM power from intra mode nonlinearity would be expected to reduce the total capacity of a fibre link by approximately $2 \mathrm{~b} / \mathrm{s} / \mathrm{Hz}$ in the limit of a high signal to noise ratio ${ }^{13}$.

\section{Acknowledgements}

This work was partly funded by the European Communities 7th Framework Programme FP/2007-2013 grant 258033 (MODE-GAP), EPSRC funded project UNLOC (grant EP/J017582/1) and Science Foundation Ireland under grant number 06/IN/I969.

\section{References}

[1] A.D.Ellis, Elect Lett, 28, pp1171 (1992).

[2] P.Poggiolini, OFC 2013, OTh3G1 (2013).

[3] X.Chen et al.,Opt.Exp,18,p19039,(2010).

[4] O.Sinkin et al., OFC 2013, OTh3G3, (2013).

[5] S.Mumtaz et al., PTL, 24, pp1574, (2012).

[6] A.Mecozzi et al,Opt.Exp,20,pp23436 (2012).

[7] A.D.Ellis, IEEE Sum. Top., FW1D1, (2012).

[8] G.Rademacher et al.,PTL,24,pp1929,(2012).

[9]R.-J.Essiambre, etal., ECOC 2012, Tu.1.C.4,

[10] R.Pini et al., Optics Letters, 9, pp79, (1984).

[11] R.-J.Essiambre,etal.,PTL,25,pp539, (2013).

[12] R.Ryf, FiO 2012, FW6.C4 (2012).

[13] A.D.Ellis et al., FiO 2012, FW1D (2012). 